\title{
Parents' perceptions of obtaining a diagnosis of childhood cancer can include experiences of disputes and delays
}

Dixon-Woods M, Findlay M, Young B, et al. Parents'accounts of obtaining a diagnosis of childhood cancer. Lancet 2001 Mar
3;357:670-4.

\section{QUESTION: What are parents' experiences of obtaining a diagnosis of cancer in their children?}

Design

Semistructured interviews.

Setting

Leicester, UK.

\section{Participants}

Parents of 20 children (age range 4-18 y) diagnosed with leukaemia, malignant solid tumour, or brain tumour, and who received treatment within the previous 4 months at a paediatric oncology unit. 4 families were of South Asian origin and the remaining families were white.

\section{Methods}

Semistructured interviews with one or both parents were held in their homes over a 7 month period. Interviews were open ended, but the interviewer used a prompt guide. Interviews were audiotaped and transcribed verbatim. Data were analysed using the constant comparison method assisted by NUD*IST software. Theoretical saturation was reached by the $14^{\text {th }}$ interview. Medical records were also analysed.

Source of funding: no external funding.

For correspondence: Dr M Dixon-Woods, Department of Epidemiology and Public Health, University of

Leicester, Leicester LE1

6TP, UK. Fax +44

(0)116252 3272.

Email:mdll@le.ac.uk.

\section{Main findings}

Parents were first alerted to their child's illness by a range of medical signs and symptoms, and by behavioural and affective cues. Parents identified 2 groups of signs and symptoms: those for which innocent explanations could be found (eg, viral infection) and those consisting of unusual or frightening events (eg, fainting). The latter group of symptoms sometimes appeared after a period of the innocent symptoms, making parents feel that investigation was urgent.
After parents decided further investigation was needed, their accounts fell into 2 categories: those with a dispute with doctors (10 families) and those with no dispute (10 families). Parents were satisfied when their child had prompt and appropriate investigation and referral, but were not satisfied if they had to insist that the doctor take action. Parents who had disputes had to argue with doctors and demand investigations, rejecting common sense diagnoses. 7 families experienced lengthy disputes, which was more likely when the cancer was difficult to diagnose. 5 families were referred to secondary care, but some parents had difficulty persuading doctors and 2 were not referred at all. Those who were referred reported long waits, which caused them distress. Disputes continued when parents had access to secondary services. Some delays ended only if parents demanded action or if a medical crisis occurred. Parents' accounts of delays were largely consistent with medical records.

Parents' reactions to the diagnosis were affected by their experiences of obtaining the diagnosis. Some parents felt vindicated or relieved that something could finally be done, whereas others were shocked, with feelings of numbness and disbelief. Some parents who had disputes with doctors felt guilty, wishing they had been more effective advocates for their child.

\section{Conclusions}

Parents of children with cancer described their experiences of obtaining a diagnosis. Half of the families experienced disputes with doctors, delays in diagnosis, and felt doctors discounted their specific knowledge of their child.

\section{COMMENTARY}

This qualitative study by Dixon-Woods et al raises several issues of direct relevance to practitioners who work with children and adolescents with cancer. Parents' knowledge about their children and their usual health patterns emerged as a crucial source of information concerning the presence of cancer. The reluctance of some doctors to take symptoms seriously may stem from the perception that childhood malignancies are rare. The conflict which then arose when parents demanded further action indicates the importance of appreciating the value of "lay" knowledge when it comes to assessing children's health. Professional knowledge is usually privileged in such situations, but this study shows that this can lead to a delay in investigations and reaching a definitive diagnosis. Such delays may then result in a lack of trust between professionals and parents, which may be difficult to recover.

The study also confirms the value of using qualitative research approaches to obtain evidence about people's detailed perceptions of healthcare services It is a good example of a practical issue being illuminated by asking people directly about their perceptions of what happened to them. Other factors that were highlighted include the dangers of medical staff lacking expertise in childhood and adolescent cancers. Recent policy changes in the UK have argued that cancer should be treated only in designated centres where the necessary skills, equipment, and experience are available. The researchers provide direct evidence why such an approach should be advocated.

The problem remains unanswered, however, about how to prevent unnecessary delays between the recognition of symptoms and cancer detection. This may require those in primary care to value a parent's instinctive knowledge of their children, so that fewer cases of cancer fall through the net. Such a message must obviously be balanced against reacting to the fears of parents who may simply be over anxious about their children. It is good to see research that focuses on the reality of cancer care and provides evidence of how a system can be improved. Similarly, research that explores adolescents' particular needs is also long overdue. Those involved in primary health and cancer care are likely to find this study thought provoking.

Daniel Kelly, RN, RNT, MSc Senior Nurse, Research and Development University College London Hospitals NHS Trust London, UK 